\title{
General anaesthesia induction using general anaesthetic agents and opioid analgesics increases Perfusion Index (PI) and decreases Pleth Variability Index (PVI): Observational clinical study
}

\author{
*Ju Mizuno, Yoshihisa Morita, Akihito Kakinuma, Shigehito Sawamura \\ Department of Anaesthesiology and the Intensive Care Unit, Teikyo University School of Medicine, \\ Tokyo 173-8605, Japan \\ *Corresponding author: mizuno_ju8@yahoo.co.jp
}

Key words: general anaesthetic agents; opioid analgesics; peripheral perfusion; pulse oximetry plethysmographic waveform; vasodilation; sympathetic tone

\begin{abstract}
Background:
General anaesthesia induction using general anaesthetic agents and opioid analgesics induces significant changes in peripheral vascular physiology and sympathetic/parasympathetic tone, thereby affecting peripheral perfusion, as observed by changes in the pulse oximetry plethysmographic waveform. Pulse CO-Oximetry ${ }^{\circledR}$ continuously and non-invasively computes Perfusion Index (PI), a measure of peripheral perfusion, and Pleth Variability Index (PVI), a measure of dynamic changes in PI during a complete respiratory cycle. In this observational clinical study, we investigated the changes in PI and PVI using Pulse COOximetry ${ }^{\circledR}$ during general anaesthesia induction.
\end{abstract}

\section{Methods:}

We prospectively recorded PI and PVI from the time before oxygen administration to that before tracheal tube or laryngeal mask insertion in 21 adult patients undergoing general anaesthesia with general anaesthetic agents and opioid analgesics for elective surgeries.

\section{Results:}

General anaesthesia induction significantly increased PI from $2.1 \pm 1.7$ to $3.8 \pm 2.3$ ( $p<$ $0.001)$ and significantly decreased PVI from $22.9 \pm 8.1$ to $17.1 \pm 7.2(p<0.05)$.

\section{Conclusion:}

PI and PVI may be useful for monitoring changes in peripheral vasodilation and sympathetic tone during general anaesthesia

\section{Introduction}

General anaesthesia induction using general anaesthetic agents and opioid analgesics cause significant changes in peripheral vascular physiology and sympathetic/parasympathetic tone. Peripheral perfusion is regulated by the autonomic nervous system under physiological conditions. General anaesthetic agents such as propofol ${ }^{1}$, desflurane $^{2}$, and isoflurane ${ }^{3}$, as well as opioid analgesics such as alfentanil ${ }^{4}$ increase the vasodilation threshold and decrease the vasoconstriction threshold by inhibiting the sympathetic nervous system. Reduction in peripheral sympathetic tone after administration of general anaesthetic agents and opioid analgesics dilates peripheral vessels, increases peripheral perfusion, and decreases arterial blood pressure. Therefore, these agents may predispose the patients to a risk of developing general anaesthesia-induced hypotension. This hypotensive response may be increased by underlying volume depletion or pre-existing cardiac dysfunction. A continuous and noninvasive method to measure a patient's sympathetic/parasympathetic response to general anaesthesia induction may help clinicians to 
identify and manage general anaesthesia-induced hypotension.

Recently, a non-invasive device, Pulse COOximetry $^{\circledR}$, has been developed that continuously detects changes in the pulse oximetry plethysmographic (POP) waveform and computes Perfusion Index (PI), a measure of peripheral perfusion, and Pleth Variability Index (PVI), a measure of dynamic changes in PI during a complete respiratory cycle, which has been shown to predict fluid responsiveness ${ }^{5,6}$.

In this observational clinical study, we investigated the changes in PI and PVI using Pulse CO-Oximetry ${ }^{\circledR}$ during general anaesthesia induction with general anaesthetic agents and opioid analgesics.

\section{Materials and Methods Study protocol}

After receiving approval from the Ethics Committee of Teikyo University School of Medicine and obtaining written informed consent, patients were enrolled for elective surgery under general anaesthesia during November 2008. The following was the inclusion criterion: adult patients with American Society of Anesthesiologists (ASA) physical status I or II. Exclusion criteria were as follows: previous known cardiac arrhythmias, intracardiac shunts, left or right ventricular dysfunction, peripheral circulation failure of the upper extremities, chronic obstructive pulmonary disease, haemodialysis, pregnancy, or inability to maintain a supine position during the study.

The patients were fasted for more than $8 \mathrm{hrs}$. After entering the operating room, an adult adhesive pulse oximeter sensor $\left(\mathrm{LNOP}^{\circledR}\right.$ Adt, Masimo Corp., Irvine, CA, USA) was placed on the index finger of each patient and wrapped using an opaque shield to prevent external light from interfering with the signal. The sensor was connected to Pulse CO-Oximetry ${ }^{\circledR}$ (Radical-7, Masimo Corp.) with SET software version 7.3.1.1. POP waveforms were recorded on a laptop computer using Satpartner ${ }^{\circledR}$ software (Masimo Corp.) and analyzed by an investigator blinded to the other haemodynamic data. An arterial pressure cuff was positioned on the arm opposite to the pulse oximeter sensor to measure systolic arterial pressure (SBP) and diastolic arterial pressure (DBP). Arterial oxygen saturation $\left(\mathrm{SpO}_{2}\right)$ and heart rate (HR) were measured using the pulse oximeter.

After oxygen was administered using a face mask for 3 mins, propofol was administered by bolus injection or using an infusion pump with a targetcontrolled infusion (TCI) system. Thereafter, the general anaesthetic agents and opioid analgesics to be administered and the method of general anaesthesia induction to be used were decided by each anaesthesiologist. After the loss of spontaneous ventilation, manual-artificial ventilation was initiated and continued. After a few minutes, the tracheal tube or laryngeal mask (LMA) was inserted. Fluid was intravenously administered at $20 \mathrm{ml} / \mathrm{kg} / \mathrm{hr}$ during general anaesthesia induction.

We prospectively recorded study data including haemodynamic and respiratory measures such as SBP, DBP, $\mathrm{SpO}_{2}$, and HR as well as PI and PVI before and after general anaesthesia induction. For the purpose of this study, "before general anaesthesia induction" was defined as the time before oxygen administration, i.e., under the room air and "after general anaesthesia induction" was defined as the time before tracheal tube or LMA insertion.

We excluded cases in which vasoactive agents, vasodilator agents, or atropine was administered during general anaesthesia induction.

\section{PI calculation}

The POP waveform relies on red and infrared light absorption, which includes two components. The first component is said to be a constant amount of light (direct current: DC), which is absorbed by skin, bone, tissue, pigments, and non-pulsatile blood. Venous blood is also responsible for some constant absorption. The second component is said to be a variable amount of light (alternating current: AC), which is absorbed by the pulsating arterial inflow. For the PI calculation, the infrared pulsatile signal is indexed against the non-pulsatile infrared signal and expressed as a percentage (PI = [AC/DC] 100), reflecting the amplitude of the POP waveform. 


\section{PVI calculation}

PVI is a measure of the dynamic change in maximum and minimum PI values over a period of time that is sufficient to include at least one complete respiratory cycle c.7. $^{5,}$ PI is then automatically and continuously calculated as $\left(\mathrm{PI}_{\max }-\mathrm{PI}_{\min }\right) / \mathrm{PI}_{\max }$, reflecting respiratory variations in PI. This algorithm allows continuous monitoring of respiratory variations in the amplitude of the POP waveform.

\section{Statistical analysis}

All data were expressed as the mean \pm standard deviation (SD) unless otherwise indicated. Changes in haemodynamic and respiratory data and PI and PVI values after general anaesthesia induction were assessed using a paired Student's $t$ test. $p<0.05$ was considered statistically significant. All statistical analyses were performed using Excel 2007 for Windows Vista (Microsoft Corp., Redmond, WA, USA).

\section{Results}

Data of 21 patients were analyzed. Subjects consisted of 11 males and 10 females aged between 25 and 75 years ( $46 \pm 15$ years). They had a mean height and body weight of $162.3 \pm 9.4$ $\mathrm{cm}$ and $61.7 \pm 13.7 \mathrm{~kg}$, respectively. Before surgery, 5 patients had taken vasodilating agents for hypertension and 1 patient had taken prostaglandin $\mathrm{E}_{1}$ for arteriosclerosis obliterans of the lower extremities. Propofol was administered by bolus injection at $1.0-2.5 \mathrm{mg} / \mathrm{kg}$ in 17 patients and using an infusion pump at a TCI concentration of $3-4 \mu \mathrm{g} / \mathrm{ml}$ in 4 patients. The patients were administered sevoflurane (9), remifentanil (6), fentanyl (12), rocuronium (16), vecuronium (2), and suxamethonium (2). Tracheal intubation and LMA insertion were performed in 19 and 2 patients, respectively. The time interval between oxygen administration and tracheal tube or LMA insertion was $7 \pm 2$ mins.

Haemodynamic and respiratory data before and after general anaesthesia induction are shown in Table 1. Following general anaesthesia induction, we observed significant decreases in SBP and DBP, a significant increase in $\mathrm{SpO}_{2}$, and no significant change in HR.
Table 1. Haemodynamic and Respiratory Data before and after general anaesthesia Induction

\begin{tabular}{|lll|}
\hline & Before & After \\
$\mathrm{SBP}(\mathrm{mmHg})$ & $142 \pm 29$ & $104 \pm 26^{* *}$ \\
$\mathrm{DBP}(\mathrm{mmHg})$ & $82 \pm 10$ & $61 \pm 16^{* *}$ \\
$\mathrm{SpO}_{2}$ & $99.0 \pm 1.2$ & $99.6 \pm 0.7^{*}$ \\
$\mathrm{HR}($ beats/min) & $75 \pm 11$ & $71 \pm 13$ \\
\hline
\end{tabular}

Mean \pm SD. $\mathrm{n}=21 . *: p<0.05$, **: $p<0.001$ vs. Before.

SBP: systolic blood pressure, DBP: diastolic blood pressure, $\mathrm{SpO}_{2}$ : arterial oxygen saturation measured using a pulse oximeter, HR: heart rate.

Moreover, we observed a significant increase in PI from $2.1 \pm 1.7 \%$ to $3.8 \pm 2.3 \%$ (Fig. 1) and a significant decrease in PVI from $22.9 \pm 8.1 \%$ to $17.1 \pm 7.2 \%$ (Fig. 2) following general anaesthesia induction.

Figure 1. Perfusion Index (PI) before and after general anaesthesia induction. $* *: p<0.001$.

Figure Legends

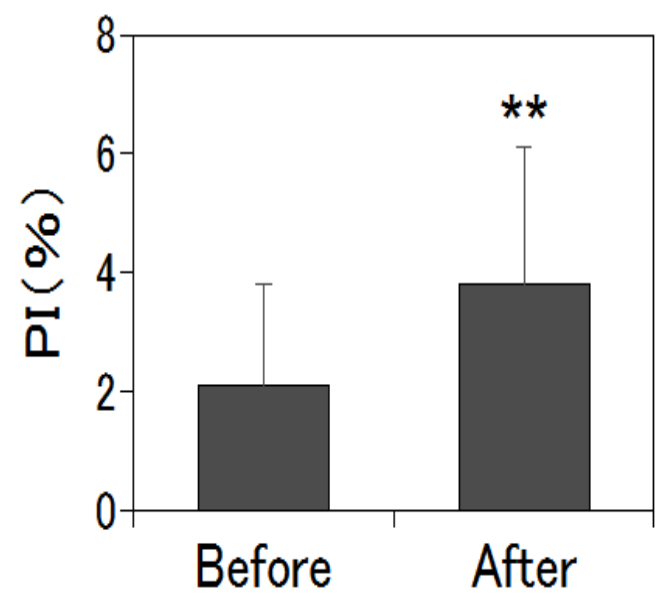

Fig.1 
Figure 2. Pleth Variability Index (PVI) before and after general anaesthesia induction. *: $p<0.05$.

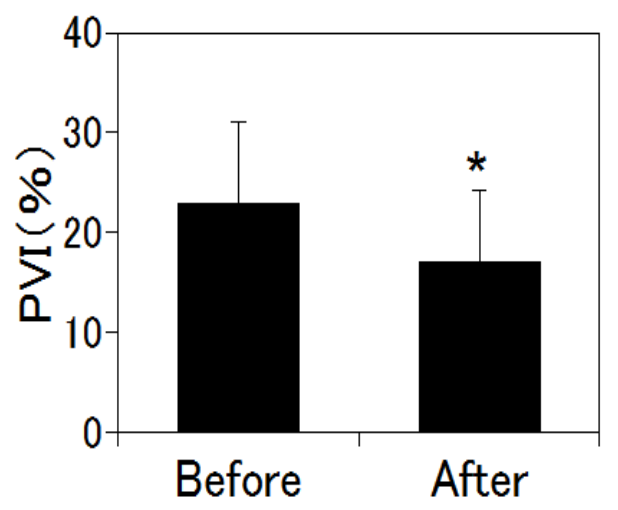

Fig.2

\section{Discussion}

Our current results demonstrate that general anesthesia induction using general anesthetic agents and opioid analgesics significantly increases PI and decreases PVI in adult patients. These findings suggest that PI and PVI monitoring indirectly detects peripheral haemodynamic changes and assesses microcirculatory effects and autonomic drives to cardiovascular target organs induced by general anaesthesia.

Administration of general anaesthetic agents causes peripheral vessels to dilate, peripheral perfusion to increase, and arterial blood pressure to decrease. For example, administration of propofol $^{8}$ or sevoflurane decreases systemic vascular resistance (SVR) in patients. Remifentanil decreases HR and arterial blood pressure by its central vagotonic effect and by stimulating peripheral $\mu$-opioid receptors in the peripheral nervous system and the cardiovascular system ${ }^{9}$. It also induces a dose-dependent increase in systemic arterial vasodilation in humans with total artificial heart implants ${ }^{10}$. Remifentanil and fentanyl induce concentration-dependent relaxation in human saphenous vein strips ${ }^{11}$ and radial artery rings ${ }^{12}$. Fentanyl, alfentanil, and sufentanil induce vasodilation by direct action on the peripheral vascular smooth muscle in the isolated canine hind limb, while increased administration causes a progressive decrease in peripheral resistance ${ }^{13}$. This hypothesis is supported by our data, which show that PI significantly increases after general anaesthesia induction. PI could be used to assess peripheral vasodilation and the increase in peripheral perfusion caused by intravenous or volatile anaesthetic agents and opioid analgesics.

PVI is a dynamic predictor of fluid responsiveness in the operating room. PVI decreases significantly with volume loading ${ }^{14}$, with the head down, i.e., in Trendelenburg ${ }^{5}$ and passive leg raising (PLR) positions ${ }^{7}$. On the other hand, PVI increases with volume depletion and with the head up, i.e., in the anti-trendelenburg position ${ }^{5}$. However, in our study, fluid was intravenously aadministered at 20 $\mathrm{ml} / \mathrm{kg} / \mathrm{hr}$ for $7 \mathrm{mins}$, i.e., approximately $140 \mathrm{ml}$ of volume loading during general anaesthesia induction. We speculate that a factor, other than fluid responsiveness, caused the decrease in PVI.

The decrease in PVI may be related to the fact that the slow oscillations induced by peripheral vasomotor tone are inhibited and only the respiratory variations appear. Under physiological conditions, peripheral perfusion is regulated by the autonomic nervous system. The anxious period before general anaesthesia induction for surgical patients may lead to increases in arterial blood pressure and sympathetic tone. PVI before general anaesthesia induction significantly correlates with a decrease in arterial blood pressure ${ }^{15}$. General anaesthetic agents and opioid analgesics increase the vasodilation threshold and decrease the vasoconstriction threshold by inhibiting the sympathetic nervous system ${ }^{1-4}$. It is suggested that PVI is susceptible to the activation of the sympathetic nervous system. Therefore, the increase in sympathetic tone might enhance PVI, whereas a decrease in sympathetic tone might reduce PVI.

In the present observational study, respiratory conditions before and after general anaesthesia induction are different. Before general anaesthesia induction the patient is spontaneously breathing, whereas after induction respiration is manually or mechanically controlled, because general anaesthetic agents and opioid analgesics inhibit not only circulation but also respiration. Under these respiratory modes, there may be respiratory variations that affect PI and PVI. PI and PVI have already been tested in anaesthetized mechanically 
ventilated patients ${ }^{5,14}$ and spontaneously breathing volunteers ${ }^{7}$. Assessment of fluid responsiveness in mechanically ventilated patients has been extensively studied, and dynamic indicators that rely on cardiopulmonary interactions are the best predictors ${ }^{16,17}$. For example, change in the amplitude of the pulse oximetry plethysmographic waveform $(\triangle \mathrm{POP})$ has been shown to be a reliable predictor of fluid responsiveness in the operating room in mechanically ventilated patients ${ }^{18-20}$. PLR mimics rapid and transient fluid loading, and these changes are reflected in changes in $\triangle \mathrm{POP}$ in spontaneously breathing volunteers ${ }^{21}$. Therefore, $\triangle \mathrm{POP}$, in association with changes in aortic blood flow, provides a useful tool to evaluate fluid responsiveness not only in mechanically ventilated but also in spontaneously breathing patients who are suspected of being hypovolemic ${ }^{22}$. However, assessment of fluid responsiveness in spontaneously breathing patients is difficult, and cardiopulmonary interactions differ greatly from those observed in mechanically ventilated patients $^{23-25}$. Tidal volumes $(\mathrm{Vt})$ and respiratory frequency (Rf) may vary breath to breath in spontaneously breathing patients. Dynamic indicators have been confirmed to be strongly influenced by the ventilator settings such as Vt, $\mathrm{Rf}$, and positive end-expiratory pressure (PEEP). Because PVI is calculated as the difference between the two extreme PI values during one complete respiratory cycle, PVI is also one of the dynamic indicators. Increase in $\mathrm{Vt}$ and PEEP induces an increase in PVI in mechanically ventilated patients under general anaesthesia ${ }^{26}$. Variations in intrathoracic pressure during spontaneous breathing might induce the variations in intravascular volume, be insufficient to modify loading conditions of the ventricles, and influence PI and PVI. In addition, hyperventilation with the change from spontaneous breathing to manual ventilation during general anaesthesia induction causes peripheral vasodilation. Therefore, we need to investigate the effect of general anaesthetic agents and opioid analgesics on PI and PVI with animal models under controlled mechanical ventilation.

The data obtained in this clinical study is not sufficient to support the use of PI and PVI monitoring during general anaesthesia induction, because a number of factors can affect the results.
The simultaneous measurement of cardiac output, peripheral perfusion, vasomotor tone, and sympathetic tone using transpulmonary thermodilution, arterial pressure-based cardiac output (APCO) devices ${ }^{27}$, pulse contour cardiac output (PiCCO) devices ${ }^{28}$, laser Doppler flowmetry ${ }^{29}$, and R-R interval analysis is required to fully understand how changes in PI and PVI, relate to the physiology of general anaesthesia induction.

In conclusion, general anaesthesia induction using general anaesthetic agents and opioid analgesics increases PI and decreases PVI, reflecting peripheral vasodilation and decreased sympathetic tone. PI and PVI could provide a useful noninvasive means of monitoring of changes in peripheral vasodilation and sympathetic tone during general anaesthesia induction.

\section{References}

1. Matsukawa T, Kurz A, Sessler DI, et al. Propofol linearly reduces the vasoconstriction and shivering thresholds. Anesthesiology 1995;82:1169-80.

2. Annadata R, Sessler DI, Tayefeh F, et al. Desflurane slightly increases the sweating threshold but produces marked, nonlinear decreases in the vasoconstriction and shivering thresholds. Anesthesiology 1995;83:1205-11.

3. Xiong J, Kurz A, Sessler DI, et al. Isoflurane produces marked and nonlinear decreases in the vasoconstriction and shivering thresholds. Anesthesiology 1996;85:240-5.

4. Kurz A, Go JC, Sessler DI, et al. Alfentanil slightly increases the sweating threshold and markedly reduces the vasoconstriction and shivering thresholds. Anesthesiology 1995;83:293-9.

5. Cannesson M, Delannoy B, Morand A, et al. Does the Pleth variability index indicate the respiratoryinduced variation in the plethysmogram and arterial pressure waveforms? Anesth Analg 2008;106:118994.

6. Loupec T, Nanadoumgar H, Frasca D, et al. Pleth variability index predicts fluid responsiveness in critically ill patients. Crit Care Med 2011;39:294-9.

7. Keller G, Cassar E, Desebbe O, et al. Ability of pleth variability index to detect hemodynamic changes induced by passive leg raising in spontaneously breathing volunteers. Crit Care 2008;12:R37.

8. Onder M, Babacan A, Bozkirli F, et al. The effect of propofol on systemic vascular resistance during cardiopulmonary bypass: a comparative study with thiopentone. Rinsho Kyobu Geka 1994;14:317-20. 
9. Shinohara K, Aono H, Unruh GK, et al. Suppressive effects of remifentanil on hemodynamics in barodenervated rabbits. Can J Anaesth 2000;47:361-6.

10. Ouattara A, Boccara G, Köckler U, et al. Remifentanil induces systemic arterial vasodilation in humans with a total artificial heart. Anesthesiology 2004;100:602-7.

11. Duman A, Saide Sahin A, Esra Atalik K, et al. The in vitro effects of remifentanil and fentanyl on isolated human right atria and saphenous veins. $J$ Cardiothorac Vasc Anesth 2003;17:465-9.

12. Gursoy S, Bagcivan I, Yildirim MK, et al. Vasorelaxant effect of opioid analgesics on the isolated human radial artery. Eur J Anaesthesiol 2006;23:496-500.

13. White DA, Reitan JA, Kien ND, Thorup SJ. Decrease in vascular resistance in the isolated canine hindlimb after graded doses of alfentanil, fentanyl, and sufentanil. Anesth Analg 1990;71:2934.

14. Cannesson M, Desebbe O, Rosamel P, et al. Pleth variability index to monitor the respiratory variations in the pulse oximeter plethysmographic waveform amplitude and predict fluid responsiveness in the operating theatre. $\mathrm{Br} \mathrm{J}$ Anaesth 2008;101:200-6.

15. Tsuchiya M, Yamada T, Asada A. Pleth variability index predicts hypotension during anesthesia induction. Acta Anaesthesiol Scand 2010;54:596602.

16. Michard F, Boussat S, Chemla D, et al. Relation between respiratory changes in arterial pulse pressure and fluid responsiveness in septic patients with acute circulatory failure. Am J Respir Crit Care Med 2000;162:134-8.

17. Solus-Biguenet H, Fleyfel M, Tavernier B, et al. Non-invasive prediction of fluid responsiveness during major hepatic surgery. $\mathrm{Br} J$ Anaesth 2006;97:808-16.

18. Cannesson M, Attof Y, Rosamel P, et al. Respiratory variations in pulse oximetry plethysmographic waveform amplitude to predict fluid responsiveness in the operating room. Anesthesiology 2007;106:1105-11.

19. Wyffels PA, Durnez PJ, Helderweirt J, et al. Ventilation-induced plethysmographic variations predict fluid responsiveness in ventilated postoperative cardiac surgery patients. Anesth Analg 2007; 105:448-52.

20. Feissel M, Teboul JL, Merlani P, et al. Plethysmographic dynamic indices predict fluid responsiveness in septic ventilated patients. Intensive Care Med 2007;33:993-9.

21. Delerme S, Renault R, Le Manach Y, et al. Variations in pulse oximetry plethysmographic waveform amplitude induced by passive leg raising in spontaneously breathing volunteers. Am J Emerg Med 2007;25:637-42.

22. Monnet X, Rienzo M, Osman D, et al. Passive leg raising predicts fluid responsiveness in the critically ill. Crit Care Med 2006;34:1402-7.

23. Lamia B, Ochagavia A, Monnet X, et al. Echocardiographic prediction of volume responsiveness in critically ill patients with spontaneously breathing activity. Intensive Care Med 2007;33:1125-32.

24. Soubrier S, Saulnier F, Hubert H, et al. Can dynamic indicators help the prediction of fluid responsiveness in spontaneously breathing critically ill patients? Intensive Care Med 2007;33:1117-24.

25. De Backer D, Pinsky MR. Can one predict fluid responsiveness in spontaneously breathing patients? Intensive Care Med 2007;33:1111-3.

26. Desebbe O, Boucau C, Farhat F, et al. The ability of pleth variability index to predict the hemodynamic effects of positive end-expiratory pressure in mechanically ventilated patients under general anesthesia. Anesth Analg 2010;110:792-8.

27. McGee WT, Horswell JL, Calderon J, et al. Validation of a continuous, arterial pressure-based cardiac output measurement: a multicenter, prospective clinical trial. Crit Care 2007;11:R105.

28. Broch O, Bein B, Gruenewald M, et al. Accuracy of the pleth variability index to predict fluid responsiveness depends on the perfusion index. Acta Anaesthesiol Scand 2011;55:686-93.

29. Landsverk SA, Hoiseth LO, Kvandal P, et al. Poor agreement between respiratory variations in pulse oximetry photoplethysmographic waveform amplitude and pulse pressure in intensive care unit patients. Anesthesiology 2008;109:849-55. 